\title{
RADIUM TECHNIQUE AT THE MEMORIAL HOSPITAL, NEW YORK.
}

By Gioacchino Failla, Physicist to the Memorial Hospital.

In my visit to the leading radium institutions in England last February, I was very favourably impressed by the quality of the work done there in this field, in spite of adverse circumstances brought about by the war. I. was also impressed by the concurrence of opinion of those working with radium regarding the desirability of greater co-operation among the different institutions and individual workers. The present paper, describing the methods of treatment which are used at the Memorial Hospital at the present time, is a step toward such co-operation.

Preparation of Radium Emanation for Therapy.--As the source of radiation we use either the emanation or the active deposit of radium, never the salt itself. The latter, dissolved in acidulated water, is kept safely in a vault, and the emanation is pumped off every morning. Owing to the decomposition of water by the radiations, the emanation, when first pumped off, is mixed with a relatively very large volume of hydrogen and oxygen. Therefore, in order to be able to confine it into the small capillary glass tubes which are used in therapy, it is necessary to separate it from most of its impurities. The process we use for purifying the emanation is the one developed by Duane and Debierne in Mme. Curie's laboratory. (1) The apparatus, however, is different. The chief feature of this method is that the separation of the emanation from its impurities is brought about by chemical means and no liquid air is required.

We seal the emanation in small glass tubes of two general sizes. The larger tubes are about $0.6 \mathrm{~mm}$. in diameter, 10 to $15 \mathrm{~mm}$. long, and contain from 100 to 250 , sometimes even 400 millicuries of emanation each, when first made. The smaller tubes are alout $0.3 \mathrm{~mm}$. in diameter, $3 \mathrm{~mm}$. long, and contain from 1 to 4 mc. of emanation each. The stronger tubes are put into silver tubes as soon as possible, while the beta and gamma ray activity is still small. These silver tubes are simple jackets, $\frac{1}{2} \mathrm{~mm}$. thick and $16 \mathrm{~mm}$. long. They are enamelled on the outside, each tube having a different colour or combination of colours, so that it may be distinguished easily from the others. The outside diameter is a little over $3 \mathrm{~mm}$. The glass tube is held in the metal jacket by means of paraffin plugs at the two ends. It can be taken out at any time by melting the paraffin.

This method of holding the glass tubes in place has been found very satisfactory. It possesses the important advantage that, since there are no caps to be screwed on and off, the work of putting the glass tubes in and taking them out requires no close attention, and can be done easily with long forceps.

(1) Duane, William, "Methods of Preparing and Using Radio-active Substances in the Treatment of Malignant Disease, and of Estimating Suitable Dosages."-Boston Medical and Surgical Journal, Vol. CLXXVII, No. 83, pp. 787-799, December 6th, 1917. 
When the gamma ray activity of the tubes has reached its equilibrium value, they are measured by means of an instrument consisting of a flat ionization chamber, a variable condenser, and a quadrant electrometer, using a null method. (1) The instrument is calibrated by means of a radium standard of $51.4 \mathrm{mg}$. of Ra. The measurements of the emanation are made with the glass tubes in the silver jackets, the additional filtration by $05 \mathrm{~mm}$. of silver being neglible in comparison to $15 \mathrm{~mm}$. or more of lead which the rays have to traverse before reaching the air in the ionization chamber. A record is kept of the colour of the tube, the amount of emanation in it, and the time of measurement. The strength of the tube at any subsequent time can be then calculated.

The smaller emanation tubes, which we call "bare tubes," to emphasise the fact that they are used without additional filters, are inserted into the

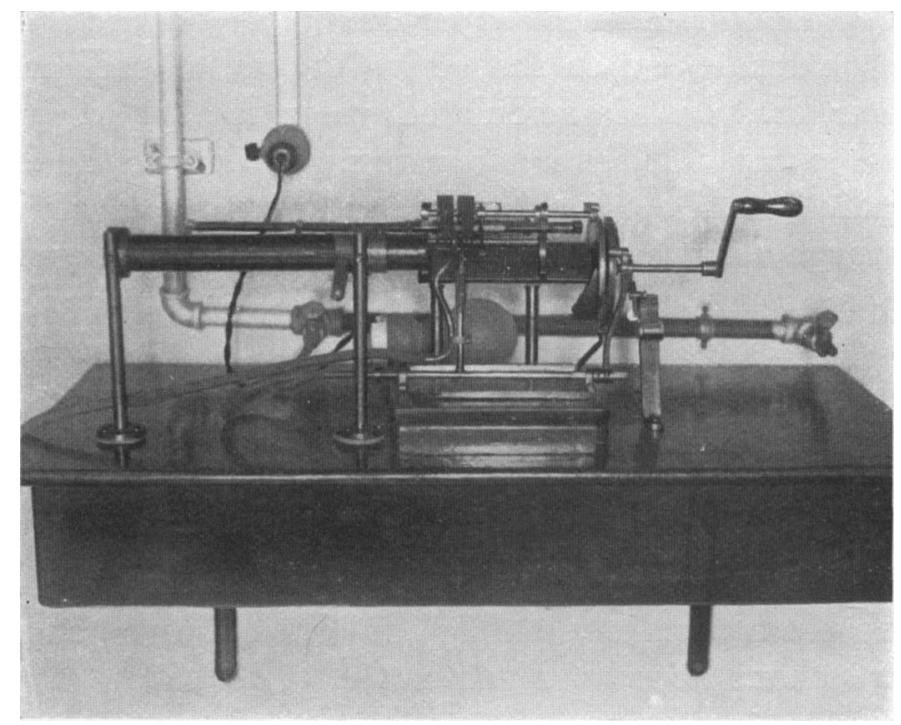

FIG. 1.-Machine used for dividing the long capillary glass tube containing the emanation into many swall tubes, $3 \mathrm{~mm}$. in length and $0.3 \mathrm{~mm}$. in diameter.

tissue. The process of making these tubes demands considerable skill on the part of the technician, and entails a long exposure of the latter to the radiations. Starting with a capillary tube about $40 \mathrm{~cm}$. long, containing 200 mc. of emanation, for example, we obtain 100 small tubes by repeated bisection by means of a small gas flame. Fach tube is then shortened to about $3 \mathrm{~mm}$. and its ends adjusted properly hy heating and removing excess glass. In the process of dividing the long capillary tube and finishing the short ones, no emanation is lost, because the pressure of the gas is considerably less than atmospheric pressure. For making these small tubes long forceps and other means of protection are provided, but they are inadequate, owing to the close attention required by such a delicate manipulation. It takes from two to three hours to make 100 of these small tubes. In this time 
the beta and gammar ray activity will have reached a value close to its maximum. The work, however, is not dangerous because a superficial beta-ray effect will manifest itself on the hands of the technician long before there is any possibility of serious injury. However, having been warned thus, the same person should not keep on doing this kind of work indefinitely. As on the average 40 to 50 tubes a day are used, it was thought desirable to develop a machine to make them. Such a machine has recently been constructed in our laboratory. Now we can make 100 tubes in less than ten minutes by simply turning a crank.

The measurement of the small amount of emanation in each tube presents some practical difficulties on account of the large number of bare tubes used in our hospital. The measuring instrument used in this case is a very sensitive galvanometer in series with a cylindrical ionization chamber containing methyl iodide. The emanation tube is dropped into a glass tube, which in turn is lowered into the ionization chamber so that the emanation is in the centre of the chamber. Under these conditions a scale deflection of nearly $4 \mathrm{~mm}$. per mc. is obtained. Measurements with this instrument can be made very rapidly. But the mere handling of such small objects takes time and requires an unlimited amount of patience.

Inasmuch as the tubes we have to measure range from 1 to $4 \mathrm{mc}$. each, naturally the accuracy of each reading is not very great. In order to minimise the effect of constant errors and to eliminate cumulative errors of calibration, the following procedure has been adopted: The tubes are put into the ionization clamber one by one and the individual readings are recorded. The tubes are then placed into a box having several compartments, those giving the same deflection being put into the same compartment. The box is then placed on the Hat ionization chamber of our standard instrument and the total amount of emanation determined, as in the case of the silver tubes. Dividing this value by the sum of the individual readings we obtain the millicuries per millimetre deflection, which, multiplied by the individual readings in millimetres, gives the amount of emanation in each tube. Since several tubes are used for one treatment and the error in each reading is just as likely to be negative as positive, the total amount of emanation used is always known with sufficient accuracy.

The same ionisation chamber and galvanometer are used for checking the values of the silver tubes just before an applicator is made up. In this way we make sure that there are no large errors in the calculated values and that no broken tubes are used over again. It is, however, very rarely that a tube is found broken. In the measurement of the silver tubes the galvanometer gives a deflection of about one millimetre per millicurie, while in the case of bare tubes one millicurie gives a deflection of nearly four millimetres.

The selection of silver as the filter is simply a matter of convenience, this metal being easy to enamel and to keep clean. The radiation which passes through $0.5 \mathrm{~mm}$. of silver is quite penetrating, but still consists mainly of beta and fairly soft gamma rays. The silver and enamel absorb about 90 per cent. 
of the radiation given off by the glass tubes, while the radiation of one of these silver tubes is reduced to half its initial value after traversing less than $2 \mathrm{~cm}$. of aluminium. This radiation is adapted for a large number of more or less superficial lesions, which form a large percentage of the cases treated in our hospital. It is for this reason that glass emanation tubes are put into silver tubes as soon as they are made. This means that the handling of the emanation consists mainly in changing the silver tubes from one applicator to another. We usually have on hand about 40 silver tubes, ranging in value from a few millicuries to about $250 \mathrm{mc}$.

In case a different filtration is desired, the glass tube can be pushed out of the silver jacket very easily after the latter has been passed rapidly over a Bunsen burner to melt the paraffin. The filters which we use are $1.5 \mathrm{~mm}$. and $1 \mathrm{~mm}$. of platinum, $2 \mathrm{~mm}$. of lead and $0.5 \mathrm{~mm}$. of silver, 0.5 and $0.2 \mathrm{~mm}$. of aluminium, or no filter at all, as in the case of the bare tubes. Other filters, such as rubber, wood, gauze, paraffin, etc., are used in making up the applicators.

The Use of Dental Compound Applicators.-The form of applicator which is chiefly responsible for the development of the enamelled silver tubes is the dental compound moulds originally used by Dr. Jaueway. These applicators are made as follows: the dental modelling compound is placed in hot water for a few minutes, after which it is quite plastic and moulds of the lesions to be treated may be made easily. The compound is applied over the lesion, moulded into the desired shape, and left in place until it has cooled and hardened. It is then taken out and the area to be treated is marked on the impression with ink. In this area deep grooves are excavated with a hot tool, care being taken not to alter the shape of the mould. Silver tubes containing the desired amount of emanation are placed into the grooves, which are then filled with paraffin. Then the applicator is placed upon the lesion and left for the proper length of time. As a rule, the treatment is not given the same day that the mould is made. Usually the impression is taken at the time the patient is examined, soon alter coming to the hospital. 'The patient's name is written on the back of the applicator and an appointment made for the treatment. Directions as to the amount of emanation desired and its distribution, protection of normal tissue, etc., are written on a special blank which accompanies the applicator.

The obvious advantage of this form of treatment is that the radio-active source is distributed over the area to be treated in exactly the way in which we desire it, and remains in the same position for the duration of the application. This is especially important in treating tongue cases because of the mobility of this organ. An applicator for a tongue lesion is usually made big enough to fill the whole mouth, and to extend between the teeth so that it may be held in place better. The use of dental compound makes it possible, also, to irradiate a tonsil at close range, by attaching the emanation tubes to a lead holder, which can be bent into the proper position, and then is held in place by fastening it to a compound mould which fits between the teeth. (See our 1916 "Radium Report.") 
The dose of radiation is specified in mc.-hours per square cm. of surface treated, stating also the total amount of emanation used and the area. The other factors are practically always constant in this form of application. The silver tubes are sunk into the mould about $3 \mathrm{~mm}$. from the surface of the impression, and they are so spaced as to have at least one tube per square $\mathrm{cm}$. of projected area. The filter is $\frac{1}{2} \mathrm{~mm}$. of silver plus 2 or $3 \mathrm{~mm}$. of paraffin and wax. The curvature of the surface covered with tubes and the area have a marked effect on the dose, because all the tubes contribute to the intensity of the radiation at any point in the tissue. Provision shonld also be made for the protection of normal tissue. When necessary we fasten, by means of paraffin, at the desired place in the compound applicator, a lead plate 2 or $3 \mathrm{~mm}$. thick, and cover the whole with paraffin. The doses, which have been found

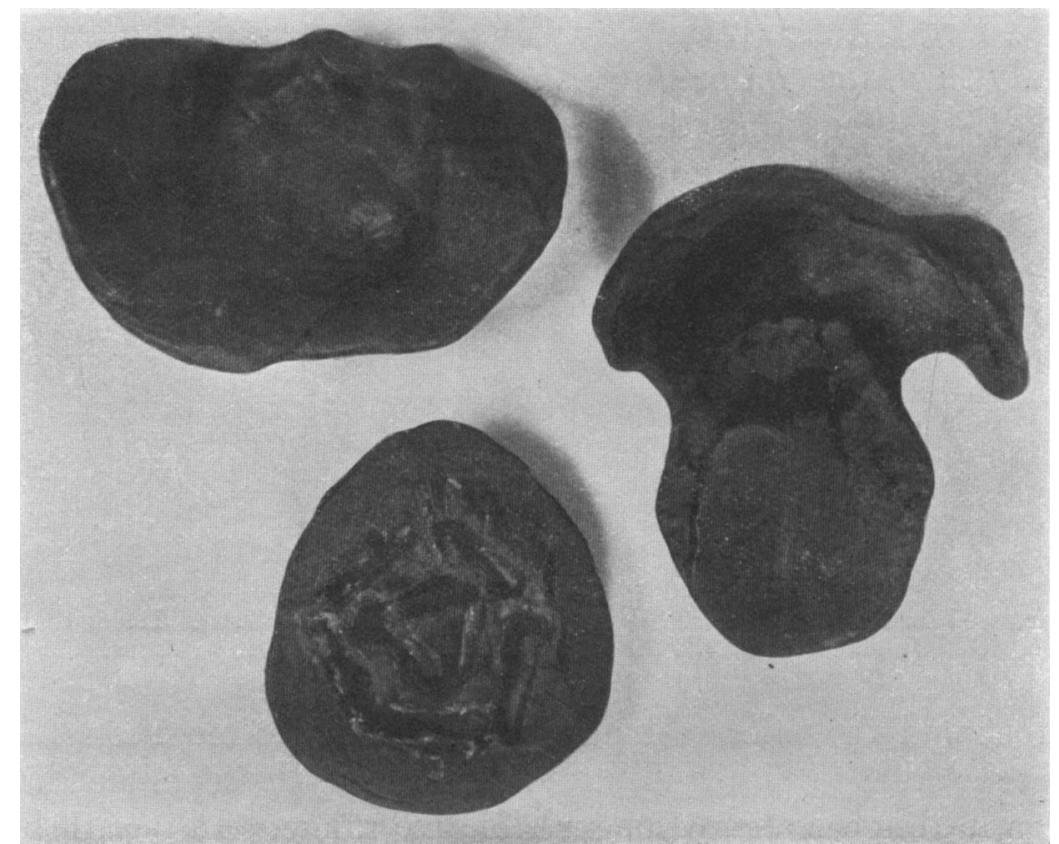

FiG. 2.-Dental modelling compuund moulds of mouth lesions. One shows the grooves after the silver tubes have been taken out.

by experience to be effective, range from 40 to $60 \mathrm{mc}$. hours per square $\mathrm{cm}$. under the conditions described above. When there is considerable cross-firing, either because the surface is large or its curvature great, the smaller dose should be given. Naturally a good deal is left to the physician in estimating the extent of the lesion, the resistivity to the radiations of the particular form of cancer, the character of the normal tissue around the cancer, and, possibly, the idiosyncrasies of the patient.

Uses of Bare Tubes.--I) luring the last two years we have treated a great many patients by inserting tiny emanation tubes into the growth by means of a steel trocar and leaving them to decay, in situ. We had previously used steel needles with a tube of emanation at the end, according to the method of Dr. Stevenson, of Dublin, with considerable success in certain cases. The 
new method, however, proved to be far more effective and of wider applicability. When the emanation is introduced into the tumour at the end of a steel needle and is withdrawn after a certain time, a relatively large amount must be used, in order that the needle shall not remain in the tissue too long. On the other hand, when the glass tubes are inserted into the tumour by means of a needle which is withdrawn immediately, leaving the emanation tube in the tissue, the amount of emanation used must be small, because its action will continue for a few weeks. Hence, we have in the first case a strong dose of radiation applied for a short time, and in the second a weak dose extended over a long interval of time. Whether the excellent results which we have obtained with this method of treatment are actually due to the difference in the intensity of the treatment, I am unable to say. Certainly it is not due to the difference in filtration in the two methods, for the quality of the radiation a short distance from the radio-active source in the two cases must be essentially the same, on account of the absorption of the radiation by the surrounding tissue itself and the small initial difference in filtration between
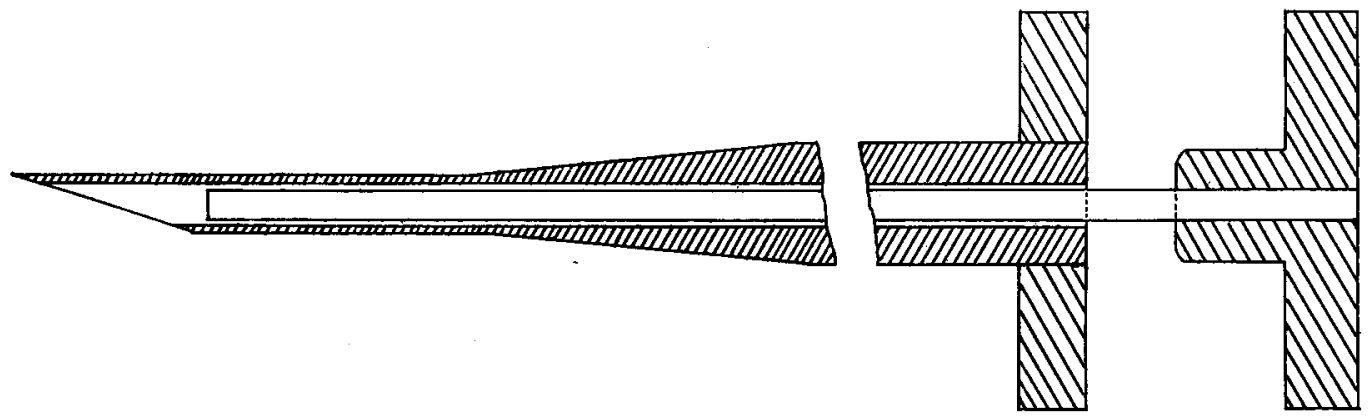

Fia. 3. -Trocar, nsed for the insertion of "bare tubes" iuto neoplastic tissue. Inside diameter, 0.6 mm. Oatside diameter, $0.8 \mathrm{~mm}$. at small end. Length, 10 to $32 \mathrm{~cm}$.

the bare tubes and the thin steel needle. It seems to me, then, that if there is any difference due to filtration, it is in favour of the steel needles, because the radiation in this case has a longer effective range. In fact, I believe that if we could use conveniently for insertion, as in the case of the bare tubes, very small tubes giving off a fairly penetrating radiation, we would obtain better results. As it is, the bare tubes are buried into the tissue with practically no discomfort to the patient; they are distributed throughout the tumour mass ; they are perfectly innocuous after the emanation has decayed, whether they slough out or not; they can be used in locations where it would be practically impossible to leave the larger steel needles for any length of time. These factors in themselves may be sufficient to explain the success of the method. It is possible that the prolonged irradiation is also a contributing factor, but this has not yet been proved experimentally.

The bare tubes are made very small in order that the trocars used for their introduction into the tissue may be as fine as possible. The latter are made of steel tubing of $0^{\circ} 6 \mathrm{~mm}$. bore, varying in length from a few $\mathrm{cm}$. to $32 \mathrm{~cm}$. The longest ones are for use through long specular. The walls of the portion 
of the needle which is inserted into the tumour are $0.1 \mathrm{~mm}$. thick, so that the perforation made in the tissue is less than $1 \mathrm{~mm}$. in diameter.

The process of embedding emanation tubes into a tumour is as follows: the piston of the trocar is withdrawn 3 or $4 \mathrm{~mm}$. and a tube of the desired strength is put at the end. The trocar is then pushed into the tumour, and when the point has reached the place where the tube is to be buried, the piston is held stationary with respect to the tumour while the needle is pulled out 3 or $4 \mathrm{~mm}$. From this point on both needle and piston can be withdrawn together. The reason for adopting this procedure is the danger of breaking the fragile glass tube if an attempt is made to drive it into the tissue by pushing the piston. The same result can be accomplished by pulling the needle as a whole out 3 or $4 \mathrm{~mm}$. and then pushing the piston so that the tube is slid into the hole previously made by the needle.

When this method of treatment was first taken up, we used to test each patient electroscopically to determine whether any tubes had been broken in the process of insertion. The patient was made to sit near an electroscope in a certain definite position, which could be duplicated easily, and a reading of the instrument taken. This was done immediately after treatment and repeated the day after. If any tubes had been broken the two readings, after making allowances for decay, would not agree. The first reading would be larger because, even though the emanation had escaped from a tube, the active deposit which was in equilibrium with the emanation, and which had not had time to decay at the time of the first measurement, still gave off beta and gamma rays and affected the electroscope. At first we were using considerably longer tubes than at present, and occasionally some were found broken. But soon we adopted the use of tubes about $3 \mathrm{~mm}$. in length, and no breakages occurred in a long series of cases tested. Consequently the electroscopic test was abandoned.

The most important points in this form of treatment are: (1) The tubes should not contain more than $4 \mathrm{mc}$. of emanation each (preferably 1 to $2 \mathrm{mc}$. or less), except in cases where excessive sloughing will not be harmful. (2) They should never be placed too near healthy tissue. (3) They should never be placed near large arteries, veins, or nerve trunks. (4) Great care should be exercised in treating lesions in proximity to bones, or in thin walled organs. (5) The total amount of emanation used depends on the size of the lesion, its location, and its pathological nature. No definite data can, of course, be given. As a rough working rule, however, we can say that the average dose should be about $0.5 \mathrm{mc}$. per cubic cm. of tumour tissue.

If we wish to specify the dose in millicurie-hours, we multiply the number of millicuries of emanation inserted by 132 , the average life of the emanation being 5.5 days, or 132 hours. This should be supplemented by a statement of the number of tubes inserted, the average strength of each tube, and the size of the lesion. In other words, the important thing to know is the number of millicuries per cubic cm. of tissue. 
Experiments on animals have been made to determine the radius of action of these tubes, and histological changes which take place at different distances from the radio-active source. They will soon be published by Dr. H. J. Bagg, probably in the Journal of Cancer Research.

In view of the fact, that in bare tube treatments the radio-active source is placed within the tumour mass, and that all the radiation (beta and gamma) passing through the glass tube is utilised, this method is more economical than any other involving the use of radium or emanation in tubes. But since most of the radiation is of the easily absorbable type, this is the local treatment par excellence. While in some cases this is a desirable feature, in others, where there is considerable infiltration by the tumour, for instance, it is advisable to combine the treatment with an irradiation by penetrating rays. For this reason bare tube treatments are often combined with external applications of emanation in silver tubes or lead containers $2 \mathrm{~mm}$. in thickness and placed at varions distances from the surface of the lesion.

Gamme Ran Applicators._-One of the applicators which we use lor deep

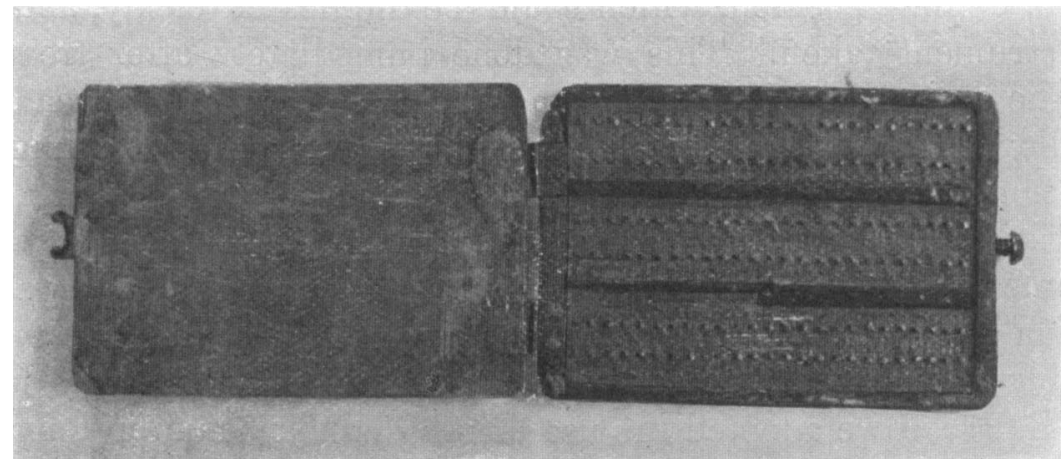

FIG. 4.- " Pack," used for external gamma-ray treatments.

radium therapy consists of a flat box, 7 by $10 \mathrm{~cm}$., in which we put a large amount of emanation distributed among many silver tubes. The bottom is lined with lead $2 \mathrm{~mm}$. thick, on which there are many rows of headless nails $4 \mathrm{~mm}$. high and $4 \mathrm{~mm}$. apart. The silver tubes fit in between the nails and are kept in place by a cover, which closes tightly over the nails. In this way as many as 72 silver tubes can be distributed evenly over this area, making up a dose of $3,000 \mathrm{mc}$. if desired. Ordinarily, however, the amount of emanation employed in this form of treatment ranges from 1,000 to $2,500 \mathrm{mc}$.

This applicator, which we call a pack, is usually placed at a distance from the skin. The distance is varied according to the depth of the tissue which we wish to affect, and ranges from 4 to $10 \mathrm{~cm}$. The pack is held in place by means of a gauze pad of the proper thickness and adhesive tape. The doses of radiation given vary from $10,000 \mathrm{mc}$. hours at a distance of $6 \mathrm{~cm}$. to 18,000 mc. hours at a distance of $10 \mathrm{~cm}$. This is close to the erythema dose for the skin. 'The ordinary device of cross-firing is resorted to whenever possible and the dose varied accordingly.

In general the pack is used for treatment of large and deep tumours. 
It is also often employed to supplement bare tube treatments. The principles involved in deep therapy are well known and need not be enumerated here. They have also been discussed in our 1916 "Report."

If we wish to give a more localised treatment with very penetrating gamma rays, we employ a dental compound mould of the lesion, on which is attached a small lead tray $2 \mathrm{~mm}$. thick. In this receptacle are placed enough silver tubes to make up the desired amount of emanation, paraffin being used to hold the tray and the tubes in place. The distance from the surface of the lesion to the lead tray may vary between 2 and $4 \mathrm{~cm}$., and is determined by the thickness of dental compound between the surface of the lesion and the tray. The radiation which reaches the lesion is, therefore, very penetrating, because in addition to $0.5 \mathrm{~mm}$. of silver and $2 \mathrm{~mm}$. of lead, the rays have to traverse a considerable thickness of dental compound. The absorbing power of the

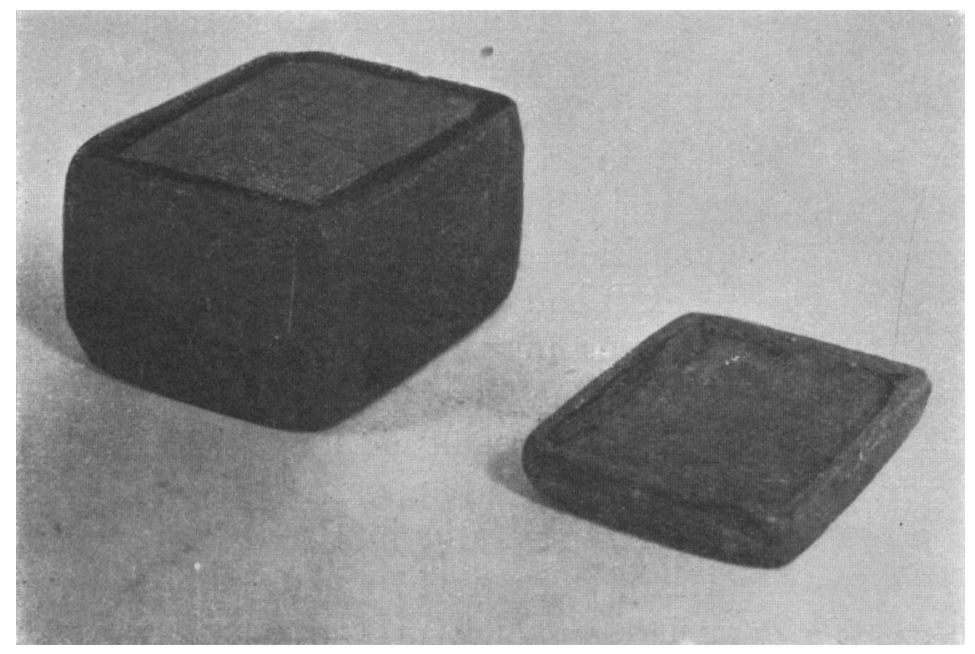

FIG. 5. - "Block" used for external gamma-ray treatments. Especially suited for cross-firing.

latter is such that $1 \mathrm{~cm}$. will reduce the radiation of a silver tube $0.5 \mathrm{~mm}$. thick by 40 per cent., and that of a lead tray $2 \mathrm{~mm}$. thick, containing silver tubes $05 \mathrm{~mm}$. thick, by 28 per cent.

Another gamma ray applicator which is used extensively consists of a block of wood, 4 by + by $6 \mathrm{~cm}$. One of the two sides, 4 by $6 \mathrm{~cm}$., is lined with lead $2 \mathrm{~mm}$. thick; the other is not. The remaining four sides are covered with lead $3 \mathrm{~mm}$. thick. The silver tubes are placed upon the $2 \mathrm{~mm}$. lead plate and are held in place by means of adhesive tape. A lead protecting cap fits over the tubes. When applied to the patient the side of the block which is not lined with lead is adjacent to the skin. 'The rays, therefore, go through $\frac{1}{2} \mathrm{~mm}$. of silver, $2 \mathrm{~mm}$. of lead and $4 \mathrm{~cm}$. of wood before reaching the skin. The wood absorbs the secondary radiation from the lead. The lead lining, $3 \mathrm{~mm}$. thick, helps to confine the radiation within the limits of the applicator. When the block is applied over neighbouring areas for the purpose of cross- 
firing it is important that overlapping of the skin area radiated be reduced as much as possible.

Special Applicators.-For the treatment of uterine cases we have been using a special applicator designed by Dr. Harold Bailey. Its construction is shown in Fig. 6 . It consists of a thin cup of iron, containing mercury to a thickness of $2 \mathrm{~cm}$. At the top of this is a lead block with a pocket for receiving the emanation tubes, which are then covered with a piece of platinum, $1 \mathrm{~mm}$. thick, and the whole held in place by a screw cap of aluminium. At

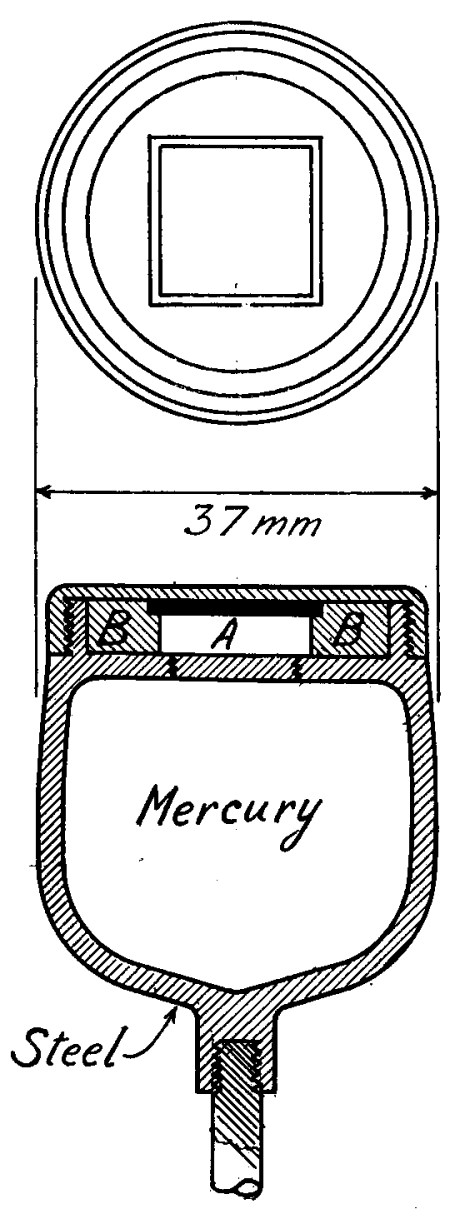

FIG. 6.-U terine applicator used by Dr. Harold Bailey. the lower end is a steel rod, about $15 \mathrm{~cm}$. long, for supporting and directing the apparatus.

The purpose of the applicator is to furnish a beam of gamma rays mainly in the forward direction, so that by pointing the device toward the part we wish to treat we can irradiate the latter without unduly affecting the surrounding tissue. However, the applicator we have been using until now does not quite fulfil these conditions. In order that we might be able to use the emanation in the silver tubes. to avoid handling the glass tubes containing large quantities of emanation, the pocket $A$ in the lead block $\mathrm{B}$ had to be made 1.8 by $1^{\circ} 6 \mathrm{~cm}$. As the outside diameter must be kept below 4 cm., the filtration sideways is not very great for the penetrating gamma rays. The result is that the intensity in the forward direction is only about 40 per cent. greater than the intensity laterally in the plane of the tubes. This probably accounts for the bladder and rectal irritation or fistule which in some cases have followed treatment with this applicator.

The protection afforded by the mercury back of the tubes is very great, the radiation in this direction being only 10 or 15 per cent. of the main beam. New applicators similar to the one here described are under construction, and it is hoped that with these the desired distribution of radiation will be obtained.

On account of the weight of such a contrivance, treatments have to be of short duration. Ordinarily we use about $1,000 \mathrm{me}$. of emanation for three hours or 3,000 mc.-hours, distributed as follows: 1,000 mc.-hours with the applicator pressing against the cervix and pointing straight ahead; 1,000 mc.-hours with the implement directed towards the left parametrium; and 1,000 mc.-hours in the direction of the right parametrium. The filtration in the forward direction is $0.5 \mathrm{~mm}$. of silver, plus $1 \mathrm{~mm}$. of platinum, plus $2 \mathrm{or}$ $3 \mathrm{~mm}$. of aluminium. The average distance of the emanation tubes from the cervix is about $1 \mathrm{~cm}$. 
To increase the accuracy of the treatment and the comfort of the patient, the applicator is supported in the proper position from the outside. For this purpose a board has been constructed with supports for the legs of the patient, and a central. support to which the stem of the applicator can be clamped in any desired position. 'This central support can be moved along the arc of a circle, so that, while the centre of the applicator remains stationary, the beam of rays can be pointed in different directions through a wide angle.

The object of the treatment with this applicator is not primarily to destroy the cancer cells in the cervix, but to reach the outposts of the disease. The local lesion can be dealt with successfully in a variety of ways. We have used steel needles $o r$ bare tubes inserted into the mass of the tumour. Also silver tubes or platinum tubes in rubber and packed with gauze against the lesion.

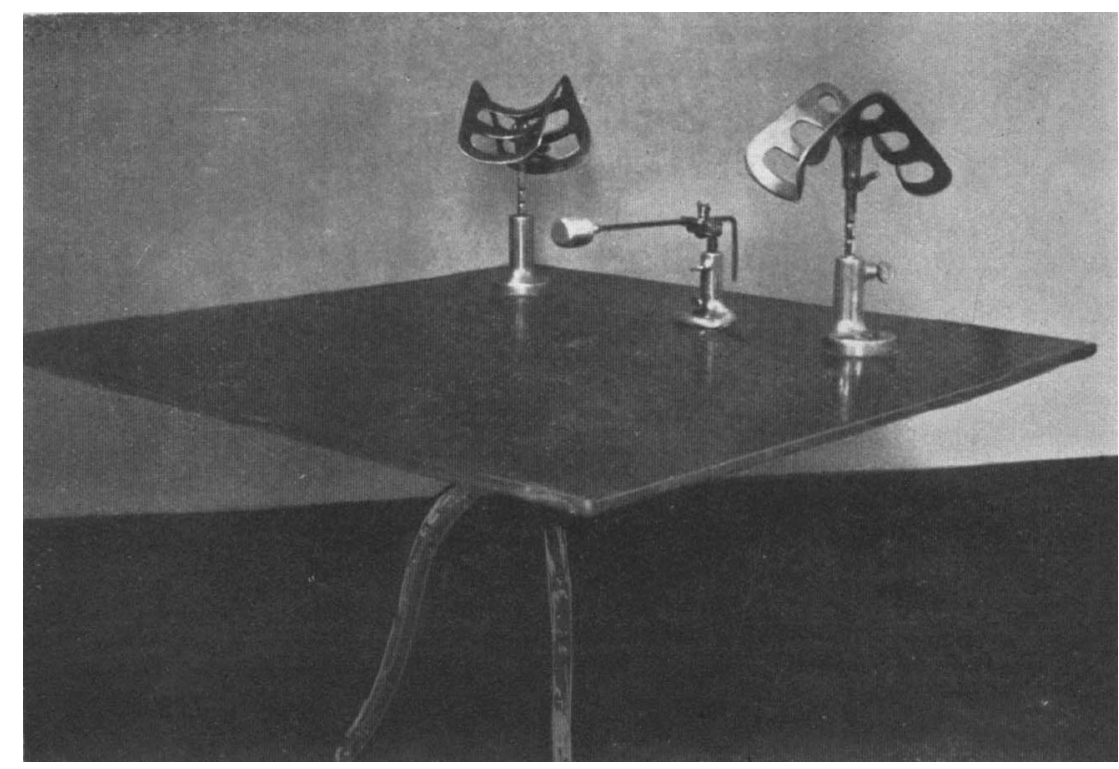

FiG. 7.-Contrivance for holding the steel-mercury uterine applicator in a fixed position with respect to the patient.

Dr. Bailey's technique for the treatment of uterine cancer is as follows : the cervical treatment consists of a dose of 1,000 to 2,000 mc.-hours, the emanation being in a platinum tube $1 \mathrm{~mm}$. thick and covered with rubber. The neck and body of the uterus are treated by inserting a similar platinum tube into the uterine canal, the dose being 1,500 mc.-hours. Sometimes a silver tube, $\frac{1}{2} \mathrm{~mm}$. thick, is used instead of the platinum tube in the uterus, and the dose is then reduced to 1,000 mc.-hours. To irradiate the parametrium and the glands within the pelvis cross-firing is resorted to. The steel-mercury applicator is used in the vagina, as already explained. The lead-lined block is used to give a total of 18,000 mc.-hours through six skin portals around the pelvis.

Dr. Janeway has been using a different method for the treatment of uterine cancer. He treats the local lesion by burying emanation tubes in it 
to the extent of 20 to $40 \mathrm{mc}$. in all. In addition he uses three platinum tubes, $1 \mathrm{~mm}$. thick, covered with rubber, in the utero-cervical canal, giving a total dose of 3,000 mc.-hours. In cases in which the fundus is involved he applies the abdominal pack at a distance of $10 \mathrm{~cm}$. from the skin, giving a dose of 20,000 me.-hours.

Both methods lead to good immediate results. Which method gives the better ultimate result, in particular as regards the percentage of recurrences, remains to be seen.

For the treatment of cancer of the bladder some sperial instruments have been designed by Dr. Benjamin S. Barringer. With these it is possible to irradiate a bladder tumour under direct observation, or to insert a steel needle, or bury "bare" tubes into it, without opening the bladder. The instruments are made of steel helices and are used in the sheath of a Brown Buerger operative cystoscope in the same way that specimen forceps are used. One has a pocket at the end to contain the strong emanation tules; the other has a suitable end and plunger for the insertion of bare tubes into the tumour.

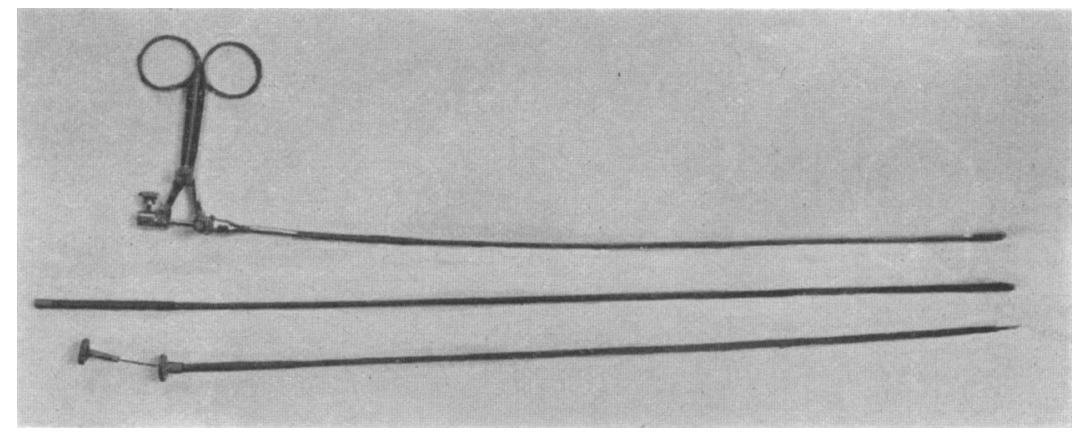

FIC. 8.-Flexible instruments for the insertion of tubes into the bladder per urethra with the aid of a cystoscope. Specimen forceps are shown for comparison of size.

In cases where the carcinoma is limited, and the limits are seen plainly through the cystoscope, the following methods have been used : (1) From 500 to 1,000 mc. of unscreened radium emanation is placed in the tip of the suitable flexible instrument. This is passed into the bladder through a cystoscope and the emanation is held against the tumour under direct observation. The largest dose given in this manner has been about $650 \mathrm{mc}$.-hours. (2) When there is no danger of perforating the bladder wall the flexible instrument may have a steel needle at the end, which is inserted into the base of the tumour and is kept in place for the required length of time. Doses of 100 mc.-hours, or $50 \mathrm{mc}$. for two. hours, have been given. (3) The same instrument, supplied with a suitable plunger, may be used to insert bare tubes into the tumour.

For tumours which are extensive, and especially for tumours around the bladder neck and base, the usual procedure is to place into the bladder, through the sheath of a cystoscope, two silver capsules, $\frac{1}{2} \mathrm{~mm}$. thick, enclosed in rubber tubes $1 \mathrm{~mm}$. thick and attached to linen threads. The cystoscope 
is withdrawn, leaving the tubes in the bladder and the threads in the urethra. The usual dose is 800 mc.-hours, or 100 mc. for 8 hours. At the end of the treatment the tube is removed through the urethra by pulling the linen thread.

Cancer of the prostate has been treated by means of steel needles inserted through the perineum and left in place for several hours, or by burying the small bare tubes in the carcinomatous nodules. The dose for the steel needles is 50 mc. for 12 hours; for the bare tube treatment it is 3 to 5 mc., divided among several tubes, which are left in the tumour mass indefinitely.

There are other special applicators which we use from time to time as occasion arises. The field of their usefulness, however, is too limited to warrant a description in the present paper.

Preparation and Uses of Active Deposit. - The name active deposit is given to the solid matter which is formed by the disintegration of the radium emanation. There are two kinds of active deposit; one, consisting of $\operatorname{Ra} \Lambda$, Ra B, and Ra C, decays very rapidly ; the other, consisting of Ra 1 ), Ra E, and Ra F, has a much longer period of disintegration. The former is called the "active deposit of rapid change," and the latter the "active deposit of slow change." The name originated from the fact that any object which is exposed to the emanation becomes "coated" with a deposit of radio-active matter. This property is made use of in the collection for our therapentic applications of the deposit. There are other means of collection, but they need not be described here.

So far we have used the active deposit in two ways; either on lead foil or on common salt $(\mathrm{NaCl})$, which is then dissolved in distilled water to give us a "radio-active solution." To collect the active deposit on lead foil, we line the inner surface of a glass tube with a piece of lead foil, about $2 \mathrm{by} 4 \mathrm{~cm}$., and $0.1 \mathrm{~mm}$. thick. The foil should cover the whole surface of the tube, and should fit snugly, so that it cammot slide down when the tube is sealed to the emanation apparatus. This having been done, the air is pumped out, and about $200 \mathrm{mc}$. of emanation is passed into the tube, the mercury being raised up to the lead foil. In three or four hours the equilibrium amount of active deposit has been formed, and the radio-activity of the foil is at a maximum. The total amount of active deposit corresponds to the amount of emanation in the tube, and is distributed over the whole surface which was exposed to the emanation. If the foil was very close to the wall of the glass tube, so that there was very little emanation in the intervening space, most of the active deposit is on the inner surface of the lead foil. Under the most favourable conditions, however, some will be deposited on the ceiling of the glass tube and on the surface of the mercury which confines the emanation in the glass tube. Therefore proper allowances must be made for loss of deposit in estimating the amount of emanation to be passed into the tube.

When the maximum amount of radio-active matter has been deposited out (three or four hours after the emanation was passed into the tube), the emanation is passed into another part of the apparatus and collected in the usual manner. The tube is cut off and the lead foil is taken out, breaking the glass if necessary. 
Its activity is then measured by means of a gamma ray instrument which has been previously calibrated.

There is no special unit for the quantity of active deposit ; for convenience we express it in millicuries, as in the case of the emanation; one millicurie being that anount of active deposit which gives the same gamma radiation through $1.5 \mathrm{~cm}$. of lead as 1 mc. of emanation or $1 \mathrm{gm}$. of radium element. When measurements are made through $1^{\circ} 5 \mathrm{~cm}$. of lead, the gamma radiation which affects the instrument is given off almost entirely by Ra C, so that whether we use a tube of radium, or the emanation, or the active deposit, in any case we really measure the amount of $\mathrm{Ra} C$ present at the time of measurement. The relative amounts of $\mathrm{Ra} \mathrm{A}$, $\mathrm{Ra} \mathrm{B}$, and $\mathrm{Ra} \mathrm{C}$ present at any subsequent time depend on the previous history of the radio-active material we are measuring. If, for example, we expose the lead foil to the emanation for one or two hours, and then measure its gamma ray activity at intervals of a few minutes, we find that at first it increases and later it decreases. The highest reading may be as much as 30 or 40 per cent. larger than the first reading.* It is evident, then, that in order to be able to duplicate our doses, we must obtain the active deposit and must, measure it always in the same manner. 'The best procedure is to expose to the emanation the lead or salt for three hours or longer, so that the active deposit is practically in equilibrium with the emanation, and it contains definite proportions of Ra $\Lambda$, Ra $P$, and Ra C. Then the curve of decay which is shown in Fig. 113, p. 497, of Rutherford's "Radio-active Substances and their Radiations," will be always the same. Furthermore, it will be possible to determine the amount of active deposit present at any time, provided we know its value at one definite time and the time when the emanation was removed from the tube containing the lead foil or salt, by referring to the above mentioned curve.

We have used the active deposit on lead foil, as here described, for the treatment of vernal catarh only. The method has been found very convenient and quite successful. The mode of application is as follows : having measured the activity of the lead foil, we determine the amount per square centimetre of surface. Then we cut a piece of the desired size and fold over it a fresh piece of lead foil (of the same thickness) so that both sides are completely covered. The corners are then cut off to prevent injury to the eye, vaseline is put on both sides, and it is inserted between the eyelid and eyeball in such a manner that the surface which was exposed to the emanation is contiguous to the part which we wish to treat. T'o reduce the disconfort of the treatment, the eye is cocainised previous to the application of the lead foil.

If the applicator is prepared as described above, the effect is due mainly to beta rays. The radiation which reaches the eyelid is filtered by $0.1 \mathrm{~mm}$. of lead, while that which falls on the eyeball is screened by $0.2 \mathrm{~mm}$. of lead. This affords sufficient protection for the latter. The vaseline adds materially to the filtration on either side of the foil. Since the distance between the radio-active source and the surface we wish to treat is rery small, the

* This is due to the different rates of decay and recovery of $\mathrm{Ra} A, \mathrm{Ra} B$, and $\mathrm{Ra} C$. 
controlling factor in the dose of radiation administered is not the total amount of active deposit used, but the surface density, i.e., the millicuries per square centimetre. We have found that a dose of 200 millicurie-minutes per square centimetre (other conditions being as described above) will cure the disease in a large majority of cases in one treatment. The duration of the treatment. should be as short as possible in order that the applicator may be taken out before the effect of the cocaine has worn off. This necessitates the use of a considerable amount of active deposit. We use letween 80 and 40 mc. per sq. $\mathrm{cm}$., so that the time varies between 5 and 10 minutes. If the application is made soon after the active deposit has been collected, its decay during the treatment is negligible.

Before leaving this subject I wish to emphasise the importance of protecting the cornea. The procedure described above, if properly carried out, insures perfect safety. Great care must be exercised, however, to make sure that no part of the foil on which the active deposit is comes in direct contact with the cornea, and also that the activated surface is facing the eyelid. The edges of the foil which were exposed to the emanation are also strongly radio-active. This fact should be borne in mind when covering the piece of the desired size with fresh lead fril. If these precautions are not taken serious burns may develop on the cornea.

The method of preparing the active deposit for purposes of injection is as follows: the bulb $\mathrm{A}$ of the tube, shown in Fig. 9, is packed tightly with salt ( $\mathrm{NaCl})$. The diameter of the bulb is small, so that only a small quantity of salt need be used, and the neck is constricted so that the salt will not fall out when the tube is sealed to the apparatus in a vertical position. The tube is heated in a gas flame to a temperature of $200^{\circ}$ C.to dry the salt. It is then sealed to the emanation apparatus and exhausted thoroughly. Concentrated emanation is passed into the tube and is confined in the bulb containing the salt by raising the mercury to the level A. After three or four hours the emanation is pumped off and collected in the usual manner, and the tube is cut off at B,

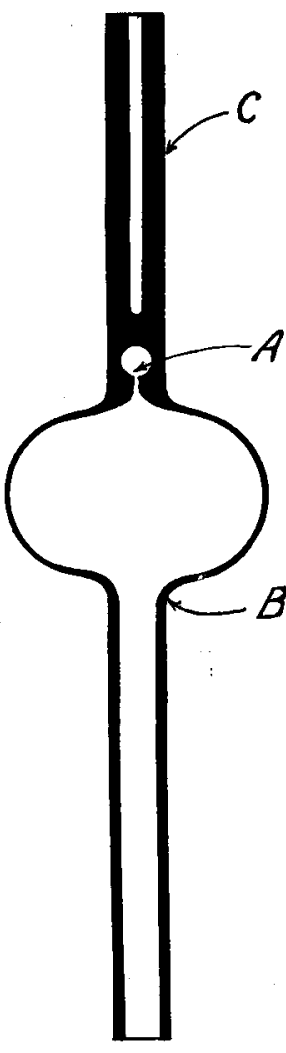

Fig 9. - Glass tube used for the collection of active deposit on salt. the tube $C$ being provided for the operator to hold it by, in order not to be too close to the active deposit. The bulb is held by a suitable pair of forceps and the needle of an hypodermic syringe inserted into the salt. Distilled water is squirted in until the large bulb is full and then sucked out again. The process is repeated a few times until all the salt is dissolved. The relative amounts of salt and water should be such as are required for a physiological solution. 'The amount of active deposit in the full syringe is determined by means of a gamma ray instrument, as in the case of the lead foil. After injecting the desired amount of solution the syringe is again measured to determine the amount of active deposit left in it. The amount actually injected 
will be the difference between the two readings, making proper allowances for decay.

Considerable difficulty is experienced in estimating the concentration of the solution, or, in other words, the amount of active deposit actually in the solution. This is due to the fact that a large fraction of it -25 to 50 per cent.adheres to the walls of the needle and syringe. In any particular case the amount which will be lost in this manner is very difficult to estimate before the injection. Consequently, it is hard to determine what amount of solution to inject in order to administer the desired dose of active deposit. However, alter having had considerable experience with the injection of active deposit, it is possible to estimate doses quite closely.

The phenomenon of the deposition of active material on the surfaces, over which the activated material is passed is of an electro-chemical nature. Accordingly, it depends on the material of the syringe, the relative areas and condition of the metal and the glass surfaces, the concentration of the solution, the time during which the solution remains in the syringe, etc. The active deposit probably is in the colloidal state, so that the presence of the sodium chloride, which is a very good electrolyte, will hasten its coagulation. But when we are dealing with such small quantities of matter it is very hard to determine just what is taking place. Experiments are being made with this end in view. For the time being, we may say that the amount of active matter which remains in the syringe may be materially reduced by passing through it some oil before the activated solution is sucked in.

The unit in which we express the amount of active deposit injected is again the millicurie. When measurements are made with a gamma ray instrument, the importance of taking into account the previous history of the active deposit is much greater in this case than in the case of the activated lead foil. As already stated, the instrument tells us the amount of $\mathrm{Ra} C$ present at the time of the measurement. However, the physiological effect of the active deposit, which is attributed to the alpha radiation, will depend not only on the amount of Ra $\mathrm{C}$ injected, but on the amount of $\mathrm{Ra} \mathrm{A}$ as well. As the decay period of the latter is much shorter than that of the former, the relative amounts of Ra $A$ and Ra $C$ present at any time after the removal of the emanation vary greatly. For instance, the number of alpha particles expelled per second by Ra $A$ and $R a C$ is the same initially, but after about 15 minutes there is no longer any $\mathrm{Ra} A$, while the radiation from the $R a \mathrm{C}$ is still very intense. It is evident, then, that if we inject the active deposit within the first 15 minutes from the time the emanation is removed from the tube containing the salt, we should take into account the radiation from Ra $A$. In our work we have injected the active deposit after all the $\mathrm{Ra} A$ had disintegrated.

The active deposit may be injected either subcutaneously or intravenously. Our work with subcutaneous injection has been limited mostly to animals.* During the last few months we have tried injecting the solution into tumours

* Some of the results are described by Dr. H. J. Bagg, in a paper which will soon appear in the Journal of Cancer Research. 
with some promising results, but it is too early yet to say anything about the merits of the treatment. On the other hand, the intravenous injection has been tried on patients more extensively, and promises to be very successful in certain diseases. We have obtained very good results in the treatment of lympathic and myologeneous leukemias, lymphosarcomas, and Hodgkin's disease by this method. The doses we have used vary from 50 to $250 \mathrm{mc}$ of active deposit: in 2 to 6 cc. of solution. It is best, perhaps, not to inject more than $200 \mathrm{mc}$. at once. Severe toxic symptoms followed the use of $250 \mathrm{mc}$. in one patient, from which, however, she recovered after a period of two months. A similar amount in other cases has not produced harmful effects.

In the preceding paragraphs I have described briefly' the principal methods of treatment which wo use at the Menorial Hospital. The description embodies those factors in the treatment which appear to the physicist to be of greatest importance. For a detailed account of the results obtained with this technique I refer the reader to the various papers published by members of the Radium Department of the hospital. A list of these papers is appended for convenience.

In closing I wish to acknowledge my indebtedness to Dr. Janeway, Dr. Bailey, Dr. Barringer, and others for their kind co-operation in the preparation of this paper.

\section{REFERFNCES.}

"The Use of Radium in Gynecological Diseases." Harold Bailey. The Amer. Journ. of Obstet. and Diseceses of Women and Children., Vol. LXXV, No. 4, 1917.

"Radium Treatment of Bladder and Prostatic Carcinoma." B. S. Barringer. The Boston Med. and Surg. Journ., Vol. CLXXVII, No. 13, $191 \%$.

"The Action of Radium on Cancer." Henry H. Janeway. Surg., Gynec. and Obstet., February, 1918.

"The Treatment of Cancer of the Lip by Radiam." Henry H. Janeway. Journ. of the Amer. Mell. Assoc., Vol. LXX, April 13, 1918.

"Treatment by Radium of Cancerous Mucous Membranes." Henry H. Janeway. Amer. Journ. of Roent., Vol. V, No. 9, September, 1918.

" Radium in Uterine Cancer." Harold Baileỳ. Surg., Gynec. and Obstet., June, 1918.

"The Treatment of Tumours of the Superior Maxilla." Henry H. Janeway. Annals of Surg., October, 1918.

"Surgery $v$. Radium in the Treatment of Carcinoma of the Bladder." Benjamin S. Barringer. New York State Journ. of Mect., November, 1918.

"Further Observations on the Radium Treatment of Uterine Cancer." Harold Bailey. The Amer. Journ. of Obstet. and Diseases of Women and Children, Vol. LXXX, No. 3, 1919.

"The Treatment of Uterine Cancer by Radium." Henry H. Janeway. Radium, Vol. XIV, No. 2, November, 1919.

"The Treatment of Malignant Tumours of the Thymus Gland by Radium." Henry H. Janeway. To appear in Annals of Surg.

"Therapentic Uses of Radium in Diseases of the Eye." Henry H. Janeway. To appear in the Archives of Ophthalm.

"The Treatment of Cancer of the Mucous Membrane by Embedded Emanation." Henry H. Janeway. To appear in the Amer. Journ, of Roent. 